\title{
DC conductivity of polyethylene and crosslinked polyethylene measured with a dynamic temperature program
}

\author{
Hossein Ghorbani \\ DONG Energy Wind Power \\ Copenhagen, Denmark \\ hosgh@dongenergy.dk
}

\author{
Carl-Olof Olsson \\ $A B B A B$, Corporate Research \\ Västerås, Sweden \\ carl-olof.olsson@se.abb.com
}

\author{
Marc Jeroense \\ NKT High Voltage Cables \\ Karlskrona, Sweden \\ marc.jeroense@nkt.com
}

\begin{abstract}
Electrical conductivity of HVDC cable insulation materials is important for its function. It is very practical to evaluate this parameter by DC conductivity measurements on press molded polymeric plates samples. While in real operation conditions, the insulation undergoes both static and dynamic thermal conditions, most of the published research in this area is still focused only on steady state thermal conditions.

In this work, the focus is instead on the behavior of electrical conductivity under dynamic thermal conditions. Press molded XLPE and LDPE plate samples with different preparations are tested under $25 \mathrm{kV} / \mathrm{mm}$ DC field with a dynamic temperature profile ranging from room temperature to $90{ }^{\circ} \mathrm{C}$.

It was discovered that in many cases, the measured conductivity during dynamic measurements strongly deviates from the expected Arrhenius temperature dependence; instead the conductivity shows a nonmonotonic temperature dependence manifested as conductivity peaks during heating and cooling. The behavior is found to be strongly related to the type of protective film used during press molding of the sample; further degassing leads to a reduction of the nonmonotonic temperature dependence and with long degassing the behavior tends to the expected Arrhenius temperature dependence.
\end{abstract}

\section{Introduction}

For HVDC insulation applications, the electric volume conductivity of a polymeric insulation material is one of its most important properties which is evaluated. The volume conductivity of the insulation is often evaluated by applying a DC voltage to press molded plaque samples and measuring the leakage current passing through the sample. In most of the published research, such measurements are performed under a constant temperature, while for practical applications the behavior of electrical properties under dynamic thermal conditions is also of interest.

In this work, the results of a series of conductivity measurements under dynamic thermal conditions are presented. The measurements are performed on press molded samples of low density polyethylene (LDPE) and crosslinked polyethylene (XLPE). The thickness of the samples is in range of $1 \mathrm{~mm}$; they are prepared using different protective pressing film and different heat treatment times. The measurements are performed with electric field of $25 \mathrm{kV} / \mathrm{mm}$ with a temperature profile, which includes both periods with constant temperature and periods with thermal cycles from room temperature up to $90^{\circ} \mathrm{C}$.

Interestingly, it was discovered that in many cases, the behavior of the measured leakage current and apparent conductivity during thermal dynamics considerably deviates from the expected Arrhenius temperature dependence; in these cases, the leakage current exhibits a non-monotonic temperature behavior with peaks appearing during heating and cooling. Further, a clear correlation was found between the types of the protective film used during the press molding of the samples.

This observation was communicated in the previous publications [1] and [2] where the observation of conductivity peaks was correlated with using polyethylene terephthalate (PET) film during the press molding of the sample. While PET film is a very common protective press film for polymeric sample preparation, there is sparingly little studies on its potential influence on the composition of the polymer samples. It has been reported that pressing LDPE samples with PET film may lead to introduction of carbonyl groups at the sample surface and strongly influences the space charge measurement results [3]. The space charge behavior of LDPE samples pressed with PET film exhibit different space charge behaviors from sample pressed with aluminum foil [2] and [4]. At higher temperatures, PET may undergo decomposition reactions forming diffusive chemicals such as acetaldehyde which may migrate into the polymer sample [5].

In this paper, this behavior is further investigated and in specific the influence protective press film and effect of heat-treatment is evaluated. The phenomenon is found to be strongly influence by the protective film and by heattreatment.

\section{DC conductivity experiments}

\subsection{Materials and sample preparation}

$1.2 \mathrm{~mm}$ thick polymeric plate samples were prepared by press molding using a Schwabenthan Polystat 300S polymer press. The polymer pellets are sandwiched between 2 layers of protective press molding film before press molding. The press molding starts with melting at 
$130{ }^{\circ} \mathrm{C}$ under 20 bar pressure for $4 \mathrm{~min}$; then the pressure is increased to $200 \mathrm{bar}$ for $2 \mathrm{~min}$ for molding, followed by crosslinking which is done at $180{ }^{\circ} \mathrm{C}$ with the same pressure for $12 \mathrm{~min}$; finally, keeping the pressure, the temperature is decreased below $35{ }^{\circ} \mathrm{C}$ before removing the sample.

To study the influence of the pressing film, some samples were prepared using PET film or aluminum foil during the whole sample preparation stages. A third series of samples were produced using PET film during melting and forming stages and polyamide (PA) film was used during the cross-linking stage.

For storage, keeping the protective pressing films, the samples were further wrapped in aluminum foil and stored in a freezer. For degassing, the protective pressing films were removed and the sample was exposed to air at $50{ }^{\circ} \mathrm{C}$ for different durations. Then the samples were again wrapped in aluminum foil and stored in freezer until measurement.

In this work 3 different HVDC grade XLPE insulations and one additive free LDPE polymer were studied.

\subsection{Experimental setup and test program}

The volume conductivity measurements were done with DC voltage using a three-electrode system made of brass; the diameter of the measurement electrode is 100 mm. A Spellman SL100*300 DC was used as voltage source and the leakage current was measured using a Keithley electrometer communicated to a computer and logged every 5 seconds. The whole electrode system was placed inside a Memmert oven to control the temperature. The air temperature in the oven was measured by the Memmert thermometer and the electrode temperature was measured using a PT100 sensor mounted on the ground electrode. More details of the measurement setup are presented in [6], [7] and [8].

Fig. 1 shows the electrode temperature during the test; the test starts at room temperature, then temperature is increased to $70{ }^{\circ} \mathrm{C}$ and kept constant for 24 hours, followed by cooling to $30^{\circ} \mathrm{C}$ during $10 \mathrm{~h}$, heating to $70^{\circ} \mathrm{C}$ during $6 \mathrm{~h}$, further heating to $90{ }^{\circ} \mathrm{C}$ during $10 \mathrm{~h}$, and finally cooling to $30^{\circ} \mathrm{C}$ during $10 \mathrm{~h}$. A DC voltage of 30 $\mathrm{kV}$ is applied during the test.

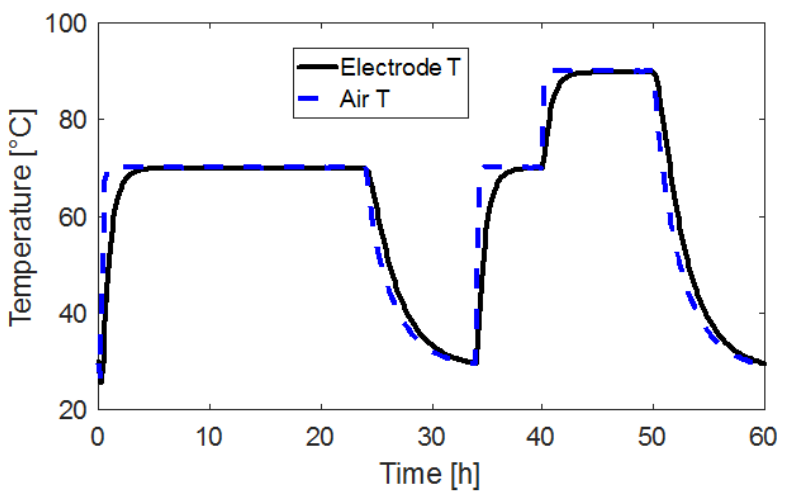

Fig. 1 - Dynamic temperature program used during the tests.

The apparent conductivity in $[\mathrm{S} / \mathrm{m}]$ is calculated from the current density $\mathrm{j}$ and the electric field $\mathrm{E}$

$$
\sigma=j /_{E}
$$

\section{Results}

\subsection{Influence of protective press films}

To study the influence of protective pressing film, the same HVDC grade XLPE insulation material was prepared either by PET film, aluminum foil and one samples were prepared using PET protective film during melting and forming and PA film for cross-linking. All samples were tested by the same test setup and with the same test program. The obtained apparent conductivity during the first $60 \mathrm{~h}$ of the test program is shown vs time in Fig. 2 and in an Arrhenius plot vs temp in Fig. 3.

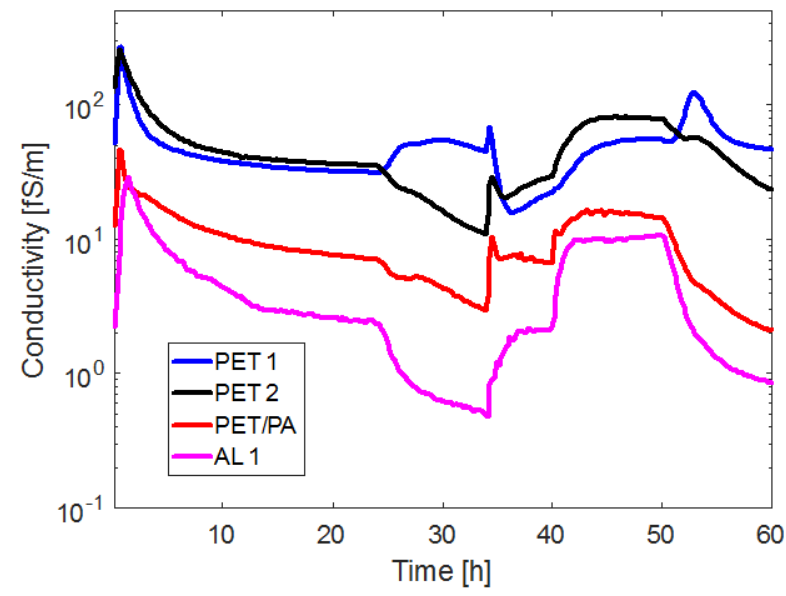

Fig. 2 - Apparent conductivity of 'XLPE A' vs time for plate samples prepared using different protective pressing films.

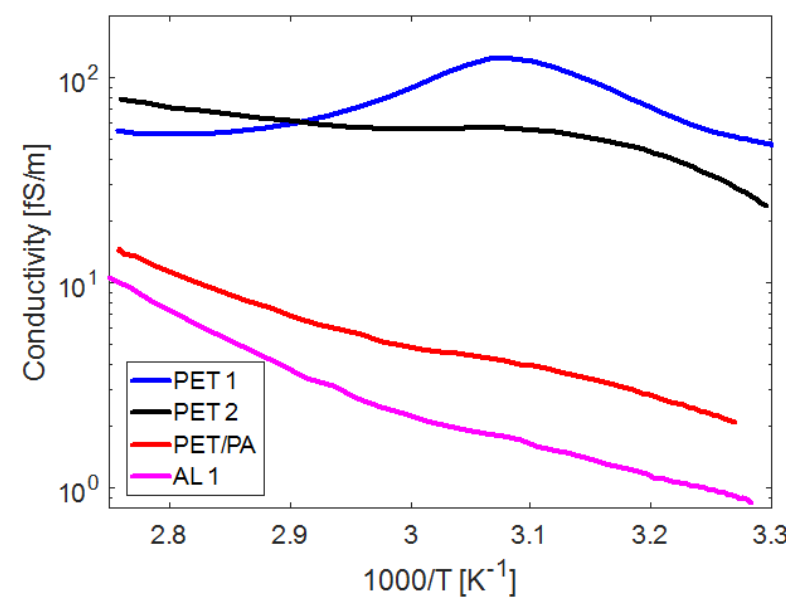

Fig. 3 - Arrhenius plot of apparent conductivity of 'XLPE A' during cooling $(50-60 \mathrm{~h})$ for plate samples prepared using different protective pressing films.

The samples prepared by PET film during the whole sample preparation show the highest apparent conductivity and the sample prepared by aluminum foil shows the lowest. Beside the conductivity level, the samples prepared by PET film show strong deviation from Arrhenius temperature dependence during cooling 
and heating periods; for these samples, a peak is observed during both heating and cooling periods. The sample which was formed by PET film and crosslinked using PA film shows an intermediate behavior.

From these results, it is obvious that the DC electrical properties of the XLPE sample is strongly influenced by the type of protective film used during press molding. It is therefore speculated that the type of protective pressing film influences the chemical composition of the samples leading to an influence on the conductivity characteristics.

\subsection{Influence of degassing}

In the next study, XLPE samples pressed with PET protective film were degassed at $50{ }^{\circ} \mathrm{C}$ by removing the protective film and hanging the samples in an air oven for different durations. The samples were then tested using the same test program as explained above.Fig. 4 presents the apparent conductivity of these samples.

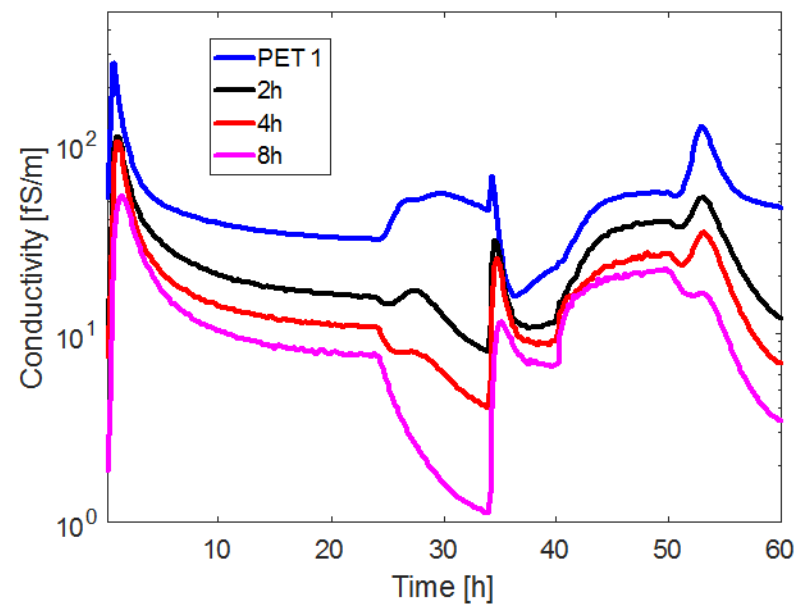

Fig. 4 - Apparent conductivity of 'XLPE A' vs time for plate samples prepared using PET protective pressing films and degassed for different durations.

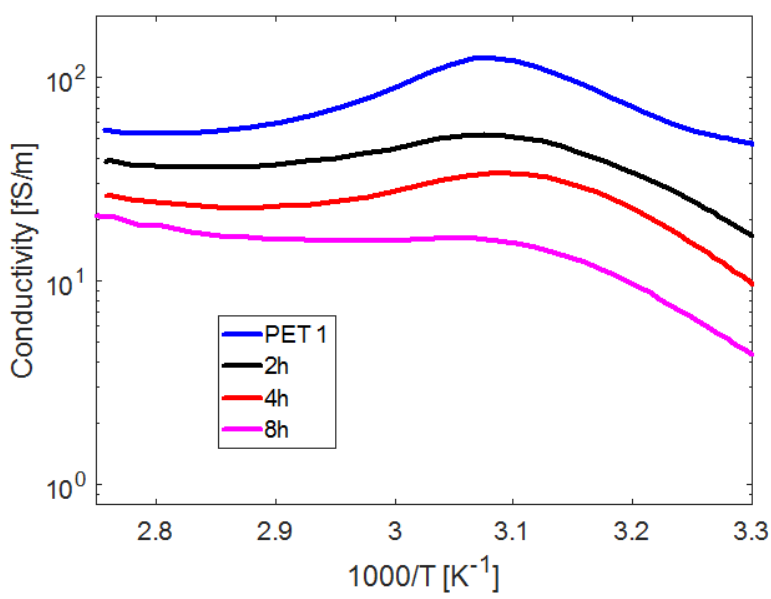

Fig. 5 - Arrhenius plot of apparent conductivity of 'XLPE A' during cooling $(50-60 \mathrm{~h})$ for plate samples prepared using PET protective pressing films and degassed for different durations.

Interestingly, a very clear trend is observed with regards to the influence of degassing; samples with longer degassing show lower conductivity and smaller peaks during heating and cooling. For sample with the longest degassing time, the conductivity behavior does not show the heating and cooling peaks and seem to tend towards the expected Arrhenius temperature dependence.

The same measurement data during the cooling 50-60 h is plotted in an Arrhenius plot in Fig. 5. In this graph, the influence of degassing on the deviation of the conductivity profile from Arrhenius behavior is clearer. As mentioned in the introduction, an Arrhenius temperature dependence will be manifested as a straight line in the Arrhenius plot. Obviously, the sample with the longest degassing time shows the closest behavior to an Arrhenius temperature dependence while the nondegassed sample strongly deviates from it and instead shows a non-monotonous temperature dependence.

From this study, it is concluded that most likely, the deviation from the Arrhenius behavior is due to some chemicals which enter the XLPE sample when they are press molded using a PET pressing film. These chemicals can be removed from the sample by degassing.

\subsection{Different insulation materials}

Fig. 6 shows the apparent conductivity of samples with different insulation materials press molded using PET pressing film. Fig. 7 shows the apparent conductivity during cooling in an Arrhenius graph. Three different HVDC grade XLPE insulation materials and one additive free LDPE insulation was used in this study.

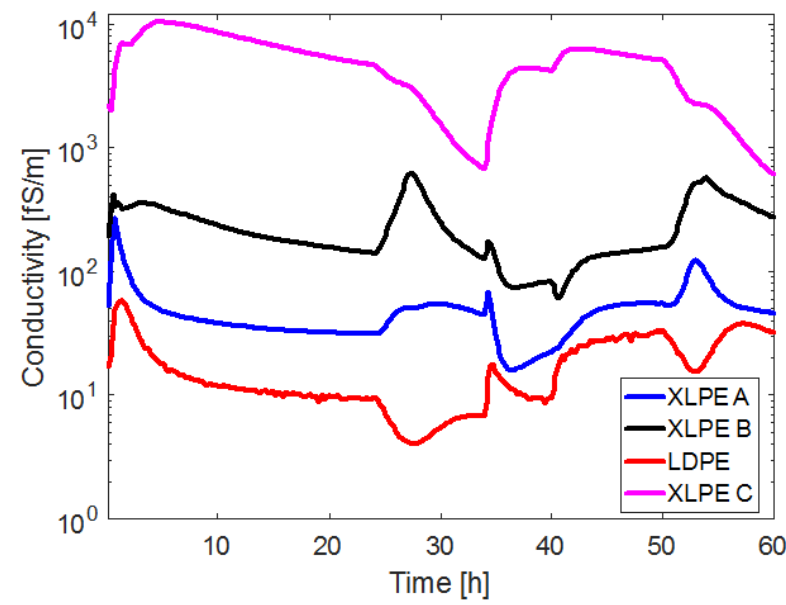

Fig. 6 - Apparent conductivity of different XLPE and LDPE insulation materials prepared using PET protective pressing film.

While different blends show different conductivity levels and LDPE shows lower conductivity than XLPE, interestingly, all samples show the peaks in the conductivity during heating and cooling. For the XLPE $\mathrm{C}$, with the highest conductivity at constant temperature, the peak during cooling and heating is less obvious. In this case, a high conductivity seems to make the peaks less clear. 


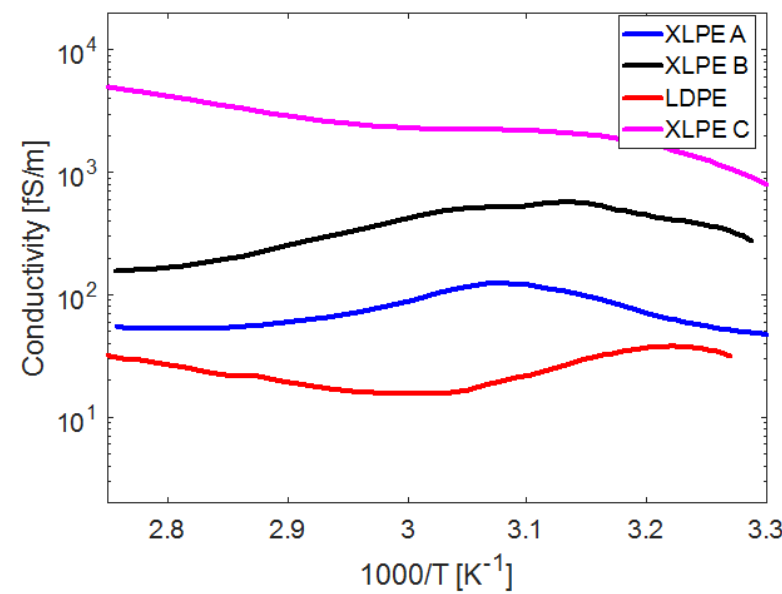

Fig. 7 - Arrhenius plot of apparent conductivity of different XLPE and LDPE insulation materials prepared using PET protective pressing film, during cooling $(50-60 \mathrm{~h})$.

This study clearly shows that the peaks during heating and cooling and deviation from Arrhenius temperature dependence are not specific to only one insulation material.

Since it is observed even in the additive free LDPE sample, this behavior is not originating from the peroxide decomposition products (PDP, also known as crosslinking by-products). This further supports the hypothesis that the peaks are related to chemicals from the PET films entering the insulation samples.

\subsection{Re-pressing using PET film}

In another study, a XLPE sample press molded using aluminum foil which did not show peaks during the conductivity measurements was sandwiched between two PET films and pressed at $180{ }^{\circ} \mathrm{C}$ for $12 \mathrm{~min}$. The measurements after this treatment, clearly showed peaks. This proves that presence of peaks is not connected to the cross-linking reactions and the peaks can be created by re-pressing a sample in contact with PET film.

\section{Conclusions}

In the previous papers, a strong deviation from Arrhenius temperature dependence of DC conductivity of XLPE and LDPE samples press molded using PET protective pressing film was reported. In this work, this interesting behavior was further investigated.

Studies on XLPE samples prepared using different aluminum and PET protective film confirms that the type of protective film has a very strong influence on the conductivity behavior of the insulation samples. Samples pressed by PET film show much higher conductivity levels than those prepared using aluminum foil. Samples formed by PET and cross-linked using Polyamide protective film show an intermediate behavior. Beside the conductivity level, sample prepared using PET protective pressing film show a strong deviation from the expected Arrhenius temperature dependence. For these samples, the dependence of conductivity on temperature seem to be non-monotonous and during cooling and heating, conductivity shows peaks.

It is speculated that this behavior is due to chemicals from the PET film entering the insulation samples during press molding.

Degassing studies confirm that these chemical species can be degassed out of the samples as longer degassing leads to both reduction of the conductivity level also a reduction in size of the peaks during cooling and heating periods. With long enough degassing time, the temperature dependence of the conductivity tends to the expected Arrhenius dependence.

Studies on three different XLPE and one additive free LDPE insulation material from two different suppliers show that these samples show the peaks during hearing and cooling when they are press molded using PET protective film. This indicates that this behavior is not specific to one insulation material or supplier instead it seems to be a more generic phenomenon.

\section{References}

[1] H. Ghorbani, M. Saltzer and C. O. Olsson, "Observation of nonmonotonic dependence of leakage current with temperature during thermal cycling", IEEE EIC 16, Montreal, Canada, 2016.

[2] H. Ghorbani, C. O. Olsson, A. Abbasi and M. Saltzer, "Influence of press films on conduction in polyethylene plaque samples", IEEE ICD 16, Montpellier, France, 2016.

[3] K. S. Suh, J.H. Koo, S. H. Lee, J. K. Park and T. Takada, "Effects of sample preparation conditions and short chains on space charge formation in LDPE," IEEE Trans. Dielec. Elec. Ins., , Vol. 3, pp. 153-160, 1996.

[4] Y. Li and T. Takada, "Space charge distribution in multi-ply LDPE," CEIDP, pp. 397-402, Oct. 18-21, 1992

[5] M. Mutsuga, Y. Kawamura, Y. Sugita-Konishi, Y. Hara-Kudo, K. Takatori and K. Tanamoto, "Migration of formaldehyde and acetaldehyde into mineral water in polyethylene terephthalate (PET) bottles", Food Addit. Contam., Vol. 23, pp. 212-218, 2006.

[6] H. Ghorbani, C. O. Olsson, J. Andersson, V. Englund, "Robust characterization of the DC-conductivity of HVDC insulation materials at high electric fields", JiCable 15, Versailles, France, 2015.

[7] C. O. Olsson, B. Källstrand, J. Ritums and M. Jeroense, "Experimental Determination of DC conductivity for XLPE Insulation," in Nordic Insul. Symp. (NORD-IS 09), Gothenburg, Sweden, 2009

[8] H. Ghorbani, "Characterization of conduction and polarization properties of HVDC cable XLPE insulation materials", Licentiate thesis report, KTH, Stockholm, 2016. 